\title{
Static Analysis of Simply Supported Functionally Graded Sandwich Plates by Using Four Variable Plate Theory
}

\author{
Pinar Aydan DEMiRHAN ${ }^{1}$ \\ Vedat TAŞKIN ${ }^{2}$
}

\begin{abstract}
In this study, the static analysis of a simply supported square functionally graded sandwich plate is performed. The core of the sandwich plate is assumed to be isotropic and the face sheets functionally graded. The variations in the effective properties of functionally graded face sheets along the thickness are obtained by using Mori-Tanaka Micromechanical Model. Four variable plate theory is used for the displacement fields. The equation of sandwich plate under sinusoidal load is obtained using the virtual displacement principle. Closed form solution is obtained with Navier's approach. Parametric values are obtained for the core and face sheet thickness ratios. The numerical results are compared with the literature and a good agreement between the obtained results and other theories in the literature is observed.
\end{abstract}

Keywords: Sandwich plate, functionally graded plate, navier's solution, bending stress, shear stress

\section{INTRODUCTION}

Laminated composite materials are very popular and attractive for many application such as space, aircraft, automotive and sports etc. Since the laminates have different material properties, stress concentrations and delamination may occur in the layer interface. In order to overcome these problems, a new composite material type whose material properties varying gradually along a function was developed. Composition and structure of this functionally graded materials change gradually along the volume as a result of the change in the properties of materials. As the application areas of functionally graded structures expand, predicting the behavior of these materials under static and dynamic loads has become an important research area. Researchers have used existing theories or proposed new ones for

Note:

- This paper has been received on February 19, 2018 and accepted for publication by the Editorial Board on October 09, 2018.

- Discussions on this paper will be accepted by May 31, 2019.

- https://dx.doi.org/10.18400/tekderg.396672

1 Trakya University, Department of Mechanical Engineering, Edirne, Turkey - pinard@trakya.edu.tr https://orcid.org/0000-0002-2618-4982

2 Trakya University, Department of Mechanical Engineering, Edirne, Turkey - vedattaskin@trakya.edu.tr https://orcid.org/0000-0002-3013-2317 
analysis of bending, buckling and vibration of functionally graded plates and beams $[1,2]$. Numerous studies using the classical plate theory $[3,4]$, which does not take into account the effects of shear deformation, the First-Order Shear Deformation Theory [3-6], which assumes shear deformation to be linear along the thickness, or High Order Shear Deformation Theories [7-16] can be seen in the literature. High Order Shear Deformation Theories can produce more accurate results, satisfying the condition where the shear stress at the boundary is zero. However, the calculating procedures of high order theories are considerably complex. Shimpi [17] developed a new theory for isotropic plates that separate transverse displacement as bending displacement and shear displacement. In addition to the computing process being very simple, the theory is accurate as a high order theory. Four Variable Plate Theory has been used by Mechab et al. [18] for the bending analysis of functionally graded materials and Houari et al. [19] for the thermoelastic bending analysis of functionally graded sandwich plates. The theory has been used in numerous studies for static and dynamic analysis of functionally graded plates due to its simplicity of calculation [20-30].

In the present study, static analysis of a square functionally graded sandwich plate is investigated by using the Four Variable Plate Theory. For the functionally graded face sheets, the effective properties of the material are varied through the thickness with a function which is derived by using Mori-Tanaka Micromechanical Model. The equations of the simply supported square plate are obtained through the virtual displacement principle. Navier's approach are used for the closed-form solution. Deflection, normal stress and shear stress values for varying ratios of core and face sheet thickness are obtained and compared with the literature.

\section{EQUATIONS OF FUNCTIONALLY GRADED SANDWICH PLATE}

A sandwich plate with a thickness of $h$, a length of $a$ and a width of $b$ is considered. For a square sandwich plate, $b$ is equal to $a$. The face sheets of the sandwich plate are assumed to be of a functionally graded material and the core is assumed to be of homogeneous isotropic material. The outer parts of the plate are metal and the inner part gradually change from metal to ceramic. The interface between face sheets and the core is completely of ceramic.

The variation of the properties of the sandwich plate along the thickness is given in Figure 1. The section between $h_{1}$ and $h_{2}$ is referred to as bottom FG face sheet, the section between $h_{2}$ and $h_{3}$ is referred to as homogeneous core and the section between $h_{3}$ and $h_{4}$ is referred to as upper FG face sheet.

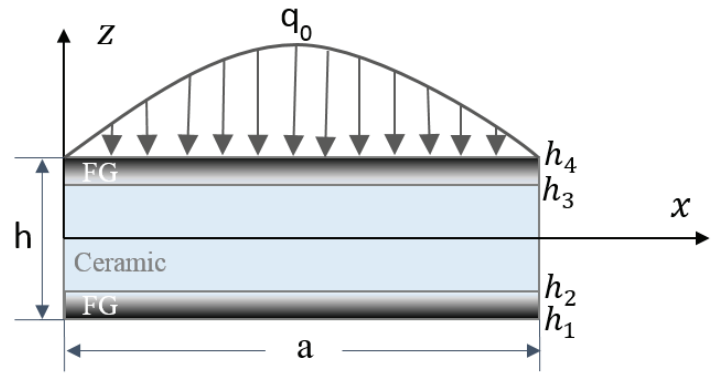

Fig. 1 - Transverse section of FG square sandwich plate with sinusoidal loading 
The variation of the elasticity modulus of the functionally graded sandwich plate is given in Equation (1). Among the lower indices, bottom is used for lower sheet and top is used for upper sheet. The lower index of $c$ represents ceramic and $m$ represents metal. $p$ is the power convention index and when it takes the value of zero, it represents the ceramic material. Increasing $p$ values represent metal-rich material. Poisson's ratio is assumed to be constant along the thickness.

$E(z)=\left\{\begin{array}{lll}E_{\text {bottom }}(z) & \text { for } & h_{1} \leq \mathrm{z} \leq \mathrm{h}_{2} \\ \mathrm{E}_{\mathrm{c}} & \text { for } & \mathrm{h}_{2}<\mathrm{z}<\mathrm{h}_{3} \\ \mathrm{E}_{\text {top }}(\mathrm{z}) & \text { for } & \mathrm{h}_{3} \leq \mathrm{z} \leq \mathrm{h}_{4}\end{array}\right.$

In Eqs. (2)-(5), $V_{c}$ and $V_{m}$ are the volume fractions of metal and ceramic materials for lower and upper face sheets.

$$
\begin{aligned}
& \mathrm{V}_{\mathrm{c}_{\text {bottom }}}(\mathrm{z})=\left(\frac{\mathrm{z}-\mathrm{h}_{1}}{\mathrm{~h}_{2}-\mathrm{h}_{1}}\right)^{\mathrm{p}} \\
& \mathrm{V}_{\mathrm{c}_{\text {top }}}(\mathrm{z})=\left(\frac{\mathrm{z}-\mathrm{h}_{4}}{\mathrm{~h}_{3}-\mathrm{h}_{4}}\right)^{\mathrm{p}} \\
& \mathrm{V}_{\mathrm{m}_{\text {bottom }}}(\mathrm{z})=1-\mathrm{V}_{\mathrm{c}_{\text {bottom }}}(\mathrm{z}) \\
& \mathrm{V}_{\mathrm{m}_{\text {top }}}(\mathrm{z})=1-\mathrm{V}_{\mathrm{c}_{\text {top }}}(\mathrm{z})
\end{aligned}
$$

In calculating the effective properties of composite materials composed of two different materials with different phases, Mori-Tanaka Micromechanical Model [30] is used. The change in the elasticity modulus of the lower and upper face sheets along the thickness is given by means of $\mathrm{E}_{\mathrm{bottom}}$ and $\mathrm{E}_{\text {top }}$ functions. Equations (6)-(7).

$$
\begin{aligned}
& E_{\text {bottom }}(z)=E_{m}+\left(E_{c}-E_{m}\right) \frac{v_{c_{b o t t o m}}(z)}{1+v_{m_{\text {bottom }}}(z)\left(\frac{E_{c}}{E_{m}}-1\right) \frac{1+v}{3(1-v)}} \\
& E_{\text {top }}(z)=E_{m}+\left(E_{c}-E_{m}\right) \frac{v_{c_{\text {top }}}(z)}{1+v_{m_{\text {top }}}(z)\left(\frac{E_{c}}{E_{m}}-1\right) \frac{1+v}{3(1-v)}}
\end{aligned}
$$

Displacement components $\mathrm{u}, \mathrm{v}$ and $\mathrm{w}$ respectively denote the displacements in the $\mathrm{x}, \mathrm{y}$, and z-axes. (Eqs. (8)-(10)) The lower index of $b$ represents the bending coupling of transverse displacement and $\mathrm{s}_{\mathrm{s}}$ represents the shear coupling of transverse displacement.

$$
\begin{aligned}
& \mathrm{u}=\mathrm{u}_{0}-\mathrm{z} \frac{\partial \mathrm{w}_{\mathrm{b}}}{\partial \mathrm{x}}+\mathrm{f}(\mathrm{z}) \frac{\partial \mathrm{w}_{\mathrm{s}}}{\partial \mathrm{x}} \\
& \mathrm{v}=\mathrm{v}_{0}-\mathrm{z} \frac{\partial \mathrm{w}_{\mathrm{b}}}{\partial \mathrm{y}}+\mathrm{f}(\mathrm{z}) \frac{\partial \mathrm{w}_{\mathrm{s}}}{\partial \mathrm{y}} \\
& \mathrm{w}=\mathrm{w}_{\mathrm{b}}+\mathrm{w}_{\mathrm{s}}
\end{aligned}
$$


Eqs. (11)-(16) give the relationships between strain and displacement.

$\varepsilon_{\mathrm{x}}=\frac{\partial \mathrm{u}}{\partial \mathrm{x}}-\mathrm{z} \frac{\partial^{2} \mathrm{w}_{\mathrm{b}}}{\partial \mathrm{x}^{2}}+\mathrm{f}(\mathrm{z}) \frac{\partial^{2} \mathrm{w}_{\mathrm{S}}}{\partial \mathrm{x}^{2}}$

$\varepsilon_{\mathrm{y}}=\frac{\partial \mathrm{v}}{\partial \mathrm{y}}-\mathrm{z} \frac{\partial^{2} \mathrm{w}_{\mathrm{b}}}{\partial \mathrm{y}^{2}}+\mathrm{f}(\mathrm{z}) \frac{\partial^{2} \mathrm{w}_{\mathrm{s}}}{\partial \mathrm{y}^{2}}$

$\varepsilon_{\mathrm{z}}=0$

$\gamma_{x y}=\frac{\partial u}{\partial y}+\frac{\partial v}{\partial x}-2 z \frac{\partial^{2} w_{b}}{\partial x \partial y}+2 f(z) \frac{\partial^{2} w_{s}}{\partial x \partial y}$

$\gamma_{\mathrm{yz}}=\mathrm{g}(\mathrm{z}) \frac{\partial \mathrm{w}_{\mathrm{s}}}{\partial \mathrm{y}}$

$\gamma_{\mathrm{xz}}=\mathrm{g}(\mathrm{z}) \frac{\partial \mathrm{w}_{\mathrm{s}}}{\partial \mathrm{x}}$

The $f(z)$ and the $g(z)$ functions are described in Equations (17)-(18).

$\mathrm{f}(\mathrm{z})=\mathrm{z}\left(\frac{1}{4}-\frac{5}{3} \frac{\mathrm{z}^{2}}{\mathrm{~h}^{2}}\right)$

$g(z)=1+\frac{d f(z)}{d z}$

The constitutive relationships for functionally graded sandwich plate can be derived as follows. (Eqs. (19)-(20).

$$
\begin{aligned}
& \left\{\begin{array}{c}
\sigma_{\mathrm{x}} \\
\sigma_{\mathrm{y}} \\
\tau_{\mathrm{xy}}
\end{array}\right\}=\left[\begin{array}{ccc}
\mathrm{Q}_{11} & \mathrm{Q}_{12} & 0 \\
\mathrm{Q}_{12} & \mathrm{Q}_{22} & 0 \\
0 & 0 & \mathrm{Q}_{66}
\end{array}\right]\left\{\begin{array}{c}
\varepsilon_{\mathrm{x}} \\
\varepsilon_{\mathrm{y}} \\
\gamma_{\mathrm{xy}}
\end{array}\right\} \\
& \left\{\begin{array}{c}
\tau_{\mathrm{yz}} \\
\tau_{\mathrm{xz}}
\end{array}\right\}=\left[\begin{array}{cc}
\mathrm{Q}_{44} & 0 \\
0 & \mathrm{Q}_{55}
\end{array}\right]\left\{\begin{array}{l}
\gamma_{\mathrm{yz}} \\
\gamma_{\mathrm{xz}}
\end{array}\right\}
\end{aligned}
$$

Rigidity expressions along the thickness can be written as follows. (Eq. (21))

$$
\begin{aligned}
& \mathrm{Q}_{11}=\mathrm{Q}_{22}=\frac{\mathrm{E}(\mathrm{z})}{1-v^{2}} \\
& \mathrm{Q}_{12}=\frac{v \mathrm{E}(\mathrm{z})}{1-v^{2}} \\
& \mathrm{Q}_{44}=\mathrm{Q}_{55}=\mathrm{Q}_{66}=\frac{\mathrm{E}(\mathrm{z})}{2(1+v)}
\end{aligned}
$$

Governing equations can be obtained by using virtual displacement principle. Where $U$ is the strain energy and $\mathrm{W}$ is the work done by external force: 


$$
\begin{aligned}
& \delta(\mathrm{U}-\mathrm{W})=0 \\
& \mathrm{U}=\int_{-\mathrm{h} / 2}^{\mathrm{h} / 2} \int_{\Omega}\left(\sigma_{\mathrm{x}} \varepsilon_{\mathrm{x}}+\sigma_{\mathrm{y}} \varepsilon_{\mathrm{y}}+\tau_{\mathrm{xy}} \gamma_{\mathrm{xy}}+\tau_{\mathrm{yz}} \gamma_{\mathrm{yz}}+\tau_{\mathrm{xz}} \gamma_{\mathrm{xz}}\right) \mathrm{d} \Omega \mathrm{dz} \\
& \mathrm{W}=\int_{\Omega} \mathrm{qwd} \Omega \\
& \delta(\mathrm{U}-\mathrm{W})=\int_{\Omega}\left[\mathrm{N}_{\mathrm{x}} \delta\left(\frac{\partial \mathrm{u}}{\partial \mathrm{x}}\right)+\mathrm{N}_{\mathrm{y}} \delta\left(\frac{\partial \mathrm{v}}{\partial \mathrm{y}}\right)+\mathrm{N}_{\mathrm{xy}} \delta\left(\frac{\partial \mathrm{u}}{\partial \mathrm{y}}+\frac{\partial \mathrm{v}}{\partial \mathrm{x}}\right)-\mathrm{M}_{\mathrm{x}}^{\mathrm{b}} \delta\left(\frac{\partial^{2} \mathrm{w}_{\mathrm{b}}}{\partial \mathrm{x}^{2}}\right)-\mathrm{M}_{\mathrm{y}}^{\mathrm{b}} \delta\left(\frac{\partial^{2} \mathrm{w}_{\mathrm{b}}}{\partial \mathrm{y}^{2}}\right)-\right. \\
& \mathrm{M}_{\mathrm{xy}}^{\mathrm{b}} \delta\left(\frac{\partial^{2} \mathrm{w}_{\mathrm{b}}}{\partial \mathrm{x} \partial \mathrm{y}}\right)-\mathrm{M}_{\mathrm{x}}^{\mathrm{s}} \delta\left(\frac{\partial^{2} \mathrm{w}_{\mathrm{s}}}{\partial \mathrm{x}^{2}}\right)-\mathrm{M}_{\mathrm{y}}^{\mathrm{s}} \delta\left(\frac{\partial^{2} \mathrm{w}_{\mathrm{s}}}{\partial \mathrm{y}^{2}}\right)-\mathrm{M}_{\mathrm{xy}}^{\mathrm{s}} \delta\left(\frac{\partial^{2} \mathrm{w}_{\mathrm{s}}}{\partial \mathrm{x} \partial \mathrm{y}}\right)+\mathrm{S}_{\mathrm{yz}}^{\mathrm{s}} \delta\left(\frac{\partial \mathrm{w}_{\mathrm{s}}}{\partial \mathrm{y}}\right)+ \\
& \left.\mathrm{S}_{\mathrm{xz}}^{\mathrm{s}} \delta\left(\frac{\partial \mathrm{w}_{\mathrm{s}}}{\partial \mathrm{x}}\right)\right] \mathrm{d} \Omega-\int_{\Omega} \mathrm{q}\left(\delta \mathrm{w}_{\mathrm{b}}+\delta \mathrm{w}_{\mathrm{s}}\right) \mathrm{d} \Omega=0
\end{aligned}
$$

Force and moment components are obtained as follows:

$$
\begin{aligned}
& N_{x}=\int_{-\frac{h}{2}}^{\frac{h}{2}} \sigma_{x} d z \\
& N_{y}=\int_{-\frac{h}{2}}^{\frac{h}{2}} \sigma_{y} d z \\
& N_{x y}=\int_{-h / 2}^{h / 2} \tau_{x y} d z \\
& M_{x}^{b}=\int_{-\frac{h}{2}}^{\frac{h}{2}} \sigma_{x} z d z \\
& M_{y}^{b}=\int_{-\frac{h}{2}}^{\frac{h}{2}} \sigma_{y} z d z \\
& M_{x y}^{b}=\int_{-\frac{h}{2}}^{\frac{h}{2}} \tau_{x y} z d z \\
& M_{x}^{s}=\int_{-\frac{h}{2}}^{\frac{h}{2}} \sigma_{x} f(z) d z \\
& M_{y}^{s}=\int_{-\frac{h}{2}}^{\frac{h}{2}} \sigma_{y} f(z) d z \\
& M_{x y}^{s}=\int_{-\frac{h}{2}}^{\frac{h}{2}} \tau_{x y} f(z) d z \\
& S_{x z}^{s}=\int_{-\frac{h}{2}}^{\frac{h}{2}} \tau_{x z} g(z) d z
\end{aligned}
$$


Static Analysis of Simply Supported Functionally Graded Sandwich Plates by ...

$$
\mathrm{S}_{\mathrm{yz}}^{\mathrm{s}}=\int_{-\mathrm{h} / 2}^{\mathrm{h} / 2} \tau_{\mathrm{yz}} \mathrm{g}(\mathrm{z}) \mathrm{dz}
$$

The rigidity matrix can be written as follows.

$$
\left\{\begin{array}{l}
\mathrm{N}_{\mathrm{x}} \\
\mathrm{N}_{\mathrm{y}} \\
\mathrm{M}_{\mathrm{x}}^{\mathrm{b}} \\
\mathrm{M}_{\mathrm{y}}^{\mathrm{b}} \\
\mathrm{M}_{\mathrm{x}}^{\mathrm{s}} \\
\mathrm{M}_{\mathrm{y}}^{\mathrm{s}}
\end{array}\right\}=\left[\begin{array}{llllll}
\mathrm{A}_{11} & \mathrm{~A}_{12} & \mathrm{~B}_{11} & \mathrm{~B}_{12} & \mathrm{~B}_{11}^{\mathrm{s}} & \mathrm{B}_{12}^{\mathrm{s}} \\
\mathrm{A}_{12} & \mathrm{~A}_{22} & \mathrm{~B}_{12} & \mathrm{~B}_{22} & \mathrm{~B}_{12}^{\mathrm{s}} & \mathrm{B}_{22}^{\mathrm{s}} \\
\mathrm{B}_{11} & \mathrm{~B}_{12} & \mathrm{D}_{11} & \mathrm{D}_{12} & \mathrm{D}_{11}^{\mathrm{s}} & \mathrm{D}_{12}^{\mathrm{s}} \\
\mathrm{B}_{12} & \mathrm{~B}_{22} & \mathrm{D}_{12} & \mathrm{D}_{22} & \mathrm{D}_{12}^{\mathrm{s}} & \mathrm{D}_{22}^{\mathrm{s}} \\
\mathrm{B}_{11}^{\mathrm{s}} & \mathrm{B}_{12}^{\mathrm{s}} & \mathrm{D}_{11}^{\mathrm{s}} & \mathrm{D}_{12}^{\mathrm{s}} & \mathrm{H}_{11}^{\mathrm{s}} & \mathrm{H}_{12}^{\mathrm{s}} \\
\mathrm{B}_{12}^{\mathrm{s}} & \mathrm{B}_{22}^{\mathrm{s}} & \mathrm{D}_{12}^{\mathrm{s}} & \mathrm{D}_{22}^{\mathrm{s}} & \mathrm{H}_{12}^{\mathrm{s}} & \mathrm{H}_{22}^{\mathrm{s}}
\end{array}\right]\left\{\begin{array}{c}
\partial \mathrm{u} / \partial \mathrm{x} \\
\partial \mathrm{v} / \partial \mathrm{y} \\
-\partial^{2} \mathrm{w}_{\mathrm{b}} / \partial \mathrm{x}^{2} \\
-\partial^{2} \mathrm{w}_{\mathrm{b}} / \partial \mathrm{y}^{2} \\
-\partial^{2} \mathrm{w}_{\mathrm{s}} / \partial \mathrm{x}^{2} \\
-\partial^{2} \mathrm{w}_{\mathrm{s}} / \partial \mathrm{y}^{2}
\end{array}\right\}
$$

The stiffness coefficients are given in Eq. (31) collectively.

$$
\left\{A_{i j}, A_{i j}^{s}, B_{i j}, B_{i j}^{s}, D_{i j}, D_{i j}^{s}, H_{i j}^{s}\right\}=\int_{-\frac{h}{2}}^{\frac{h}{2}}\left\{1, g, z, f, z^{2}, f z, f^{2}\right\} Q_{i j} d z \quad(i, j=1,2,4,5,6)
$$

Eqs. (32)-(35) are plate equilibrium equations.

$$
\begin{aligned}
& \frac{\partial N_{x}}{\partial x}+\frac{\partial N_{x y}}{\partial y}=0 \\
& \frac{\partial N_{x y}}{\partial x}+\frac{\partial N_{y}}{\partial y}=0 \\
& \frac{\partial^{2} M_{x}^{b}}{\partial x^{2}}+2 \frac{\partial M_{x y}^{b}}{\partial x \partial y}+\frac{\partial^{2} M_{y}^{b}}{\partial y^{2}}+q=0 \\
& \frac{\partial^{2} M_{x}^{S}}{\partial x^{2}}+2 \frac{\partial M_{x y}^{s}}{\partial x \partial y}+\frac{\partial^{2} M_{y}^{s}}{\partial y^{2}}+\frac{\partial N_{x z}^{s}}{\partial x}+\frac{\partial N_{y z}^{s}}{\partial y}+q=0
\end{aligned}
$$

The differential equations of the plate (Eqs. (36)-(39)), are obtained by using the force and moment components in Eq.(30) by inserting in Eqs. (32)-(35).

$$
\begin{aligned}
& \mathrm{A}_{11} \frac{\partial^{2} \mathrm{u}}{\partial \mathrm{x}^{2}}+\mathrm{A}_{66} \frac{\partial^{2} \mathrm{u}}{\partial \mathrm{y}^{2}}+\left(\mathrm{A}_{12}+\mathrm{A}_{66}\right) \frac{\partial^{2} \mathrm{v}}{\partial \mathrm{x} \partial \mathrm{y}}-\mathrm{B}_{11} \frac{\partial^{3} \mathrm{w}_{\mathrm{b}}}{\partial \mathrm{x}^{3}}-\left(\mathrm{B}_{12}+2 \mathrm{~B}_{66}\right) \frac{\partial^{3} \mathrm{w}_{\mathrm{b}}}{\partial \mathrm{x} \partial \mathrm{y}^{2}}-\mathrm{B}_{11}^{\mathrm{s}} \frac{\partial^{3} \mathrm{w}_{\mathrm{s}}}{\partial \mathrm{x}^{3}}- \\
& \left(\mathrm{B}_{12}^{\mathrm{s}}+2 \mathrm{~B}_{66}^{\mathrm{s}}\right) \frac{\partial^{3} \mathrm{w}_{\mathrm{s}}}{\partial \mathrm{x} \partial \mathrm{y}^{2}}=0 \\
& \left(\mathrm{~A}_{12}+\mathrm{A}_{66}\right) \frac{\partial^{2} \mathrm{u}}{\partial \mathrm{x} \partial \mathrm{y}}+\mathrm{A}_{66} \frac{\partial^{2} \mathrm{v}}{\partial \mathrm{x}^{2}}+\mathrm{A}_{22} \frac{\partial^{2} \mathrm{v}}{\partial \mathrm{y}^{2}}-\left(\mathrm{B}_{12}+2 \mathrm{~B}_{66}\right) \frac{\partial^{3} \mathrm{w}_{\mathrm{b}}}{\partial \mathrm{x}^{2} \partial \mathrm{y}}-\mathrm{B}_{22} \frac{\partial^{3} \mathrm{w}_{\mathrm{b}}}{\partial \mathrm{y}^{3}}-\left(\mathrm{B}_{12}^{\mathrm{s}}+\right. \\
& \left.2 \mathrm{~B}_{66}^{\mathrm{s}}\right) \frac{\partial^{3} \mathrm{w}_{\mathrm{s}}}{\partial \mathrm{x}^{2} \partial \mathrm{y}}-\mathrm{B}_{22}^{\mathrm{s}} \frac{\partial^{3} \mathrm{w}_{\mathrm{s}}}{\partial \mathrm{y}^{3}}=0 \\
& \mathrm{~B}_{11} \frac{\partial^{3} \mathrm{u}}{\partial \mathrm{x}^{3}}+\left(\mathrm{B}_{12}+2 \mathrm{~B}_{66}\right) \frac{\partial^{3} \mathrm{u}}{\partial \mathrm{x} \partial \mathrm{y}^{2}}+\left(\mathrm{B}_{12}+2 \mathrm{~B}_{66}\right) \frac{\partial^{3} \mathrm{v}}{\partial \mathrm{x}^{2} \partial \mathrm{y}}+\mathrm{B}_{22} \frac{\partial^{3} \mathrm{v}}{\partial \mathrm{y}^{3}}-\mathrm{D}_{11} \frac{\partial^{4} \mathrm{w}_{\mathrm{b}}}{\partial \mathrm{x}^{4}}-2\left(\mathrm{D}_{12}+\right. \\
& \left.2 \mathrm{D}_{66}\right) \frac{\partial^{4} \mathrm{w}_{\mathrm{b}}}{\partial \mathrm{x}^{2} \partial \mathrm{y}^{2}}-\mathrm{D}_{22} \frac{\partial^{4} \mathrm{w}_{\mathrm{b}}}{\partial \mathrm{y}^{4}}-\mathrm{D}_{11}^{\mathrm{s}} \frac{\partial^{4} \mathrm{w}_{\mathrm{s}}}{\partial \mathrm{x}^{4}}-2\left(\mathrm{D}_{12}^{\mathrm{s}}+2 \mathrm{D}_{66}^{\mathrm{s}}\right) \frac{\partial^{4} \mathrm{w}_{\mathrm{s}}}{\partial \mathrm{x}^{2} \partial \mathrm{y}^{2}}-\mathrm{D}_{22}^{\mathrm{s}} \frac{\partial^{4} \mathrm{w}_{\mathrm{s}}}{\partial \mathrm{y}^{4}}+\mathrm{q}=0
\end{aligned}
$$


$\mathrm{B}_{11}^{\mathrm{s}} \frac{\partial^{3} \mathrm{w}_{\mathrm{s}}}{\partial \mathrm{x}^{3}}+\left(\mathrm{B}_{12}^{\mathrm{s}}+2 \mathrm{~B}_{66}^{\mathrm{s}}\right) \frac{\partial^{3} \mathrm{u}}{\partial \mathrm{x} \partial \mathrm{y}^{2}}+\mathrm{B}_{22}^{\mathrm{s}} \frac{\partial^{3} \mathrm{v}}{\partial \mathrm{y}^{3}}-\mathrm{D}_{11}^{\mathrm{s}} \frac{\partial^{4} \mathrm{w}_{\mathrm{b}}}{\partial \mathrm{x}^{4}}-\mathrm{D}_{22}^{\mathrm{s}} \frac{\partial^{4} \mathrm{w}_{\mathrm{b}}}{\partial \mathrm{y}^{4}}+\left(\mathrm{B}_{12}^{\mathrm{s}}+2 \mathrm{~B}_{66}^{\mathrm{s}}\right) \frac{\partial^{3} \mathrm{u}}{\partial \mathrm{x}^{2} \partial \mathrm{y}}-$ $\mathrm{H}_{11}^{\mathrm{s}} \frac{\partial^{4} \mathrm{w}_{\mathrm{s}}}{\partial \mathrm{x}^{4}}-\mathrm{H}_{22}^{\mathrm{s}} \frac{\partial^{4} \mathrm{w}_{\mathrm{s}}}{\partial \mathrm{y}^{4}}-2\left(\mathrm{D}_{12}^{\mathrm{s}}+2 \mathrm{D}_{66}^{\mathrm{s}}\right) \frac{\partial^{4} \mathrm{w}_{\mathrm{b}}}{\partial \mathrm{x}^{2} \partial \mathrm{y}^{2}}-2\left(\mathrm{H}_{12}^{\mathrm{s}}+2 \mathrm{H}_{66}^{\mathrm{s}}\right) \frac{\partial^{4} \mathrm{w}_{\mathrm{s}}}{\partial \mathrm{x}^{2} \partial \mathrm{y}^{2}}+\mathrm{A}_{55}^{\mathrm{s}} \frac{\partial^{2} \mathrm{w}_{\mathrm{s}}}{\partial \mathrm{x}^{2}}+$ $\mathrm{A}_{44}^{\mathrm{s}} \frac{\partial^{2} \mathrm{w}_{\mathrm{s}}}{\partial \mathrm{y}^{2}}+\mathrm{q}=0$

Boundary conditions for the simply supported functionally graded sandwich plate are given in Eq.(40).

$$
\begin{aligned}
& v(0, y)=w_{b}(0, y)=w_{s}(0, y)=\frac{\partial w_{b}}{\partial y}(0, y)=\frac{\partial w_{s}}{\partial y}(0, y)=0 \\
& v(a, y)=w_{b}(a, y)=w_{s}(a, y)=\frac{\partial w_{b}}{\partial y}(a, y)=\frac{\partial w_{s}}{\partial y}(a, y)=0 \\
& N_{x}(0, y)=M_{x}^{b}(0, y)=M_{x}^{s}(0, y)=N_{x}(a, y)=M_{x}^{b}(a, y)=M_{x}^{s}(a, y)=0 \\
& u(x, 0)=w_{b}(x, 0)=w_{s}(x, 0)=\frac{\partial w_{b}}{\partial x}(x, 0)=\frac{\partial w_{s}}{\partial x}(x, 0)=0 \\
& u(x, b)=w_{b}(x, b)=w_{s}(x, b)=\frac{\partial w_{b}}{\partial x}(x, b)=\frac{\partial w_{s}}{\partial x}(x, b)=0 \\
& N_{y}(x, 0)=M_{y}^{b}(x, 0)=M_{y}^{s}(x, 0)=N_{y}(x, b)=M_{y}^{b}(x, b)=M_{y}^{s}(x, b)=0
\end{aligned}
$$

For the analytical solution of the simply supported plate, Navier's procedure is used. The external force applied is written in double series expansion according to the Navier's approach.

$\mathrm{q}(\mathrm{x}, \mathrm{y})=\sum_{\mathrm{m}=1}^{\infty} \sum_{\mathrm{n}=1}^{\infty} \mathrm{q}_{\mathrm{mn}} \sin (\alpha \mathrm{x}) \sin (\beta \mathrm{y})$

where $\alpha=\frac{m \pi}{a}, \beta=\frac{n \pi}{b}$. For sinusoidal loading, $m, n$, and $\mathrm{q}_{\mathrm{mn}}$ are defined as follows.

$\mathrm{m}=\mathrm{n}=1, \quad \mathrm{q}_{11}=\mathrm{q}_{0}$

The displacement components fulfilling the boundary conditions according to the Navier's solution procedure are considered to be as follows:

$$
\left\{\begin{array}{c}
\mathrm{u} \\
\mathrm{v} \\
\mathrm{w}_{\mathrm{b}} \\
\mathrm{w}_{\mathrm{s}}
\end{array}\right\}=\sum_{\mathrm{m}=1}^{\infty} \sum_{\mathrm{n}=1}^{\infty}\left\{\begin{array}{c}
\mathrm{U}_{\mathrm{mn}} \cos (\alpha \mathrm{x}) \sin (\beta \mathrm{y}) \\
\mathrm{V}_{\mathrm{mn}} \sin (\alpha \mathrm{x}) \cos (\beta \mathrm{y}) \\
\mathrm{W}_{\mathrm{bmn}} \sin (\alpha \mathrm{x}) \sin (\beta \mathrm{y}) \\
\mathrm{W}_{\mathrm{smn}} \sin (\alpha \mathrm{x}) \sin (\beta \mathrm{y})
\end{array}\right\}
$$

$\mathrm{U}_{\mathrm{mn}}, \mathrm{V}_{\mathrm{mn}}, \mathrm{W}_{\mathrm{bmn}}, \mathrm{W}_{\mathrm{smn}}$ are unknown coefficients. When $\mathrm{K}$ is the coefficients matrix and $\mathrm{F}$ is the applied external force, the unknown coefficients are obtained by solving the Eq. (44).

$\mathrm{K} \Delta=\mathrm{F}$ 


$$
\begin{aligned}
& \Delta^{\mathrm{T}}=\left\{\mathrm{U}_{\mathrm{mn}}, \mathrm{V}_{\mathrm{mn}}, \mathrm{W}_{\mathrm{bmn}}, \mathrm{W}_{\mathrm{smn}}\right\} \\
& \mathrm{F}^{\mathrm{T}}=\left\{\begin{array}{llll}
0, & 0,-\mathrm{q}_{\mathrm{mn}}, & -\mathrm{q}_{\mathrm{mn}}
\end{array}\right\} \\
& \mathrm{K}=\left[\begin{array}{llll}
\mathrm{K}_{11} & \mathrm{~K}_{12} & \mathrm{~K}_{13} & \mathrm{~K}_{14} \\
\mathrm{~K}_{12} & \mathrm{~K}_{22} & \mathrm{~K}_{23} & \mathrm{~K}_{24} \\
\mathrm{~K}_{13} & \mathrm{~K}_{23} & \mathrm{~K}_{33} & \mathrm{~K}_{34} \\
\mathrm{~K}_{14} & \mathrm{~K}_{24} & \mathrm{~K}_{34} & \mathrm{~K}_{44}
\end{array}\right]
\end{aligned}
$$

the coefficients of $\mathrm{K}$ matrix are given in Eq. (48)

$$
\begin{aligned}
& \mathrm{K}_{11}=\mathrm{A}_{11} \alpha^{2}+\mathrm{A}_{66} \beta^{2} \\
& \mathrm{~K}_{12}=\alpha \beta\left(\mathrm{A}_{12}+\mathrm{A}_{66}\right) \\
& \mathrm{K}_{13}=-\alpha\left[\mathrm{B}_{11} \alpha^{2}+\left(\mathrm{B}_{12}+2 \mathrm{~B}_{66}\right) \beta^{2}\right] \\
& \mathrm{K}_{14}=-\alpha\left[\mathrm{B}_{11}^{\mathrm{s}} \alpha^{2}+\left(\mathrm{B}_{12}^{\mathrm{s}}+2 \mathrm{~B}_{66}^{\mathrm{s}}\right) \beta^{2}\right] \\
& \mathrm{K}_{22}=\mathrm{A}_{66} \alpha^{2}+\mathrm{A}_{22} \beta^{2} \\
& \mathrm{~K}_{23}=-\beta\left[\left(\mathrm{B}_{12}+2 \mathrm{~B}_{66}\right) \alpha^{2}+\mathrm{B}_{22} \beta^{2}\right] \\
& \mathrm{K}_{24}=-\beta\left[\left(\mathrm{B}_{12}^{\mathrm{s}}+2 \mathrm{~B}_{66}^{\mathrm{s}}\right) \alpha^{2}+\mathrm{B}_{22}^{\mathrm{s}} \beta^{2}\right] \\
& \mathrm{K}_{33}=\mathrm{D}_{11} \alpha^{4}+2\left(\mathrm{D}_{12}+2 \mathrm{D}_{66}\right) \alpha^{2} \beta^{2}+\mathrm{D}_{22} \beta^{4} \\
& \mathrm{~K}_{34}=\mathrm{D}_{11}^{\mathrm{s}} \alpha^{4}+2\left(\mathrm{D}_{12}^{\mathrm{s}}+2 \mathrm{D}_{66}^{\mathrm{s}}\right) \alpha^{2} \beta^{2}+\mathrm{D}_{22}^{\mathrm{s}} \beta^{4} \\
& \mathrm{~K}_{44}=\mathrm{H}_{11}^{\mathrm{s}} \alpha^{4}+2\left(\mathrm{H}_{12}^{\mathrm{s}}+2 \mathrm{H}_{66}^{\mathrm{s}}\right) \alpha^{2} \beta^{2}+\mathrm{H}_{22}^{\mathrm{s}} \beta^{4}+\mathrm{A}_{55}^{\mathrm{s}} \alpha^{2}+\mathrm{A}_{44}^{\mathrm{s}} \beta^{2}
\end{aligned}
$$

\section{RESULTS}

Some results are presented for the Navier's solution of the functionally graded square sandwich plate which is under sinusoidally distributed load and simply supported in all edges. The properties of the material used for obtaining the numerical results are defined as follows:

$$
\begin{array}{lll}
\text { Ceramic }\left(\mathrm{ZrO}_{2}\right) & \mathrm{E}_{\mathrm{c}}=151 \mathrm{GPa}, & v=0.3 \\
\operatorname{Metal}(\mathrm{Al}) & \mathrm{E}_{\mathrm{m}}=70 \mathrm{GPa}, & v=0.3
\end{array}
$$

In order to compare the values with those in the literature, the following non-dimensional expressions are used.

$$
\bar{W}=\frac{10 E_{0}}{q_{0} a^{2}} W\left(\frac{a}{2}, \frac{b}{2}\right), \overline{\sigma_{x}}=\frac{10 h^{2}}{q_{0} a^{2}} \sigma_{x}\left(\frac{a}{2}, \frac{b}{2}, \frac{h}{2}\right), \overline{\tau_{x z}}=\frac{h}{q_{0} a} \tau_{x z}\left(0, \frac{b}{2}, 0\right)
$$

where $\mathrm{E}_{0}=1 \mathrm{GPa}$. For the comparison of numerical results, plate thickness ratios found in the literature are used. Four symmetric and two non-symmetric ratios are used.

(1-0-1) FG sandwich plate: The plate without a core. $\mathrm{h}_{2}=0$ and $\mathrm{h}_{3}=0$ 
(1-1-1) FG sandwich plate: The thickness of the core and face sheets are equal. $h_{2}=-h / 6$ and $\mathrm{h}_{3}=\mathrm{h} / 6$

(1-2-1) FG sandwich plate: The functionally graded face sheets have half the thickness of the core. $h_{2}=-h / 4$ and $h_{3}=h / 4$

(2-1-2) FG sandwich plate: The core has half the thickness of the functionally graded face sheets. $h_{2}=-h / 10$ and $h_{3}=h / 10$

(2-1-1) FG sandwich plate: The thickness of the lower face sheet is twice as much as the thickness of the core and upper face sheet. $h_{2}=0$ and $h_{3}=h / 4$

(2-2-1) FG sandwich plate: The upper face sheet has half the thickness of the core and lower face sheet $h_{2}=-h / 10$ and $h_{3}=3 h / 4$

The results are presented in the Tables 1-3 and in the Figs. 3-7.

In the Figure 2, the variation of elasticity modulus along the thickness for symmetric and non-symmetric sandwich plates is given. The plate, which is metal in the outer surfaces, turns into a ceramic-rich form as it gets closer to the midplane. The change in the elasticity modulus diverges from being linear with the increasing power law index and shows parabolic distribution.

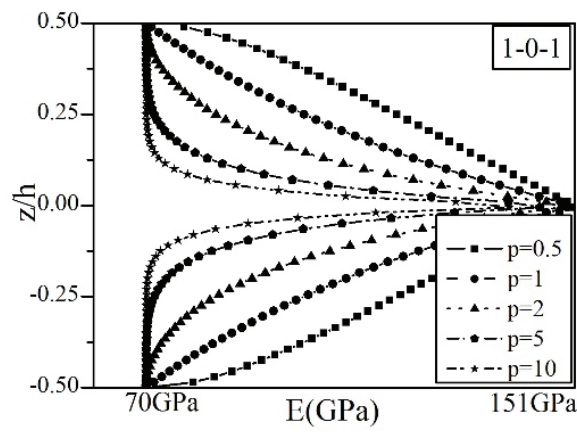

(a) (1-0-1) FG sandwich plate

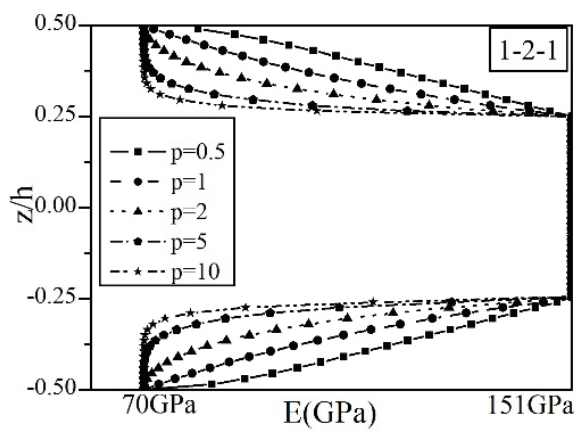

(c) (1-2-1) FG sandwich plate

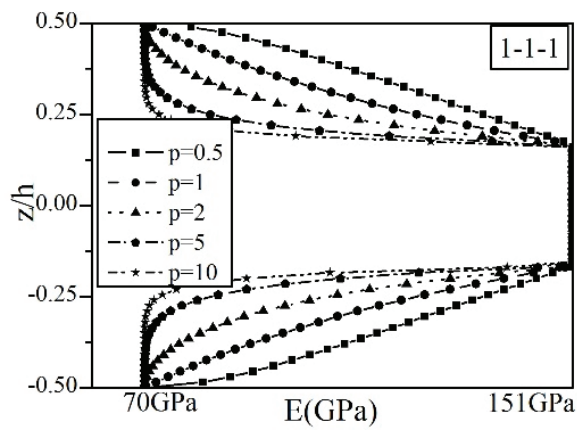

(b) (1-1-1) FG sandwich plate

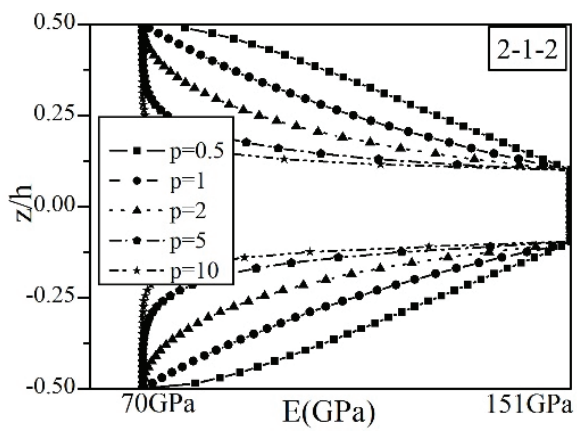

(d) (2-1-2) FG sandwich plate 


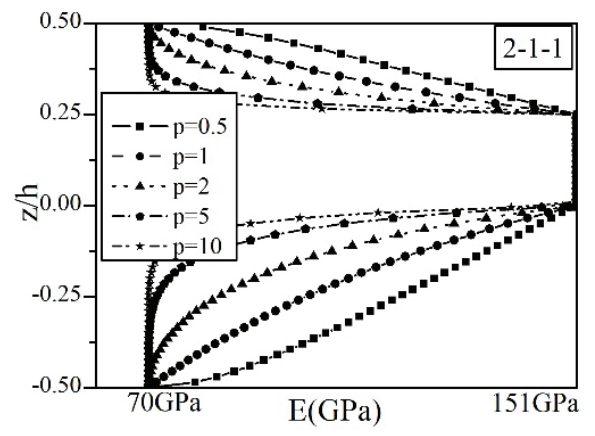

(e) (2-1-1) FG sandwich plate

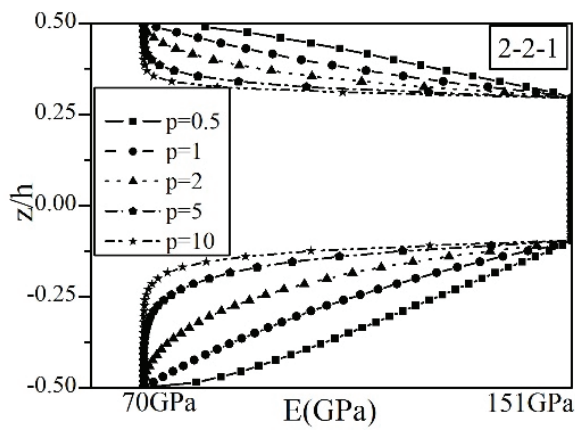

(f) (2-2-1) FG sandwich plate

Fig.2 - Elasticity modulus function with different $p$ values $(E c=151 \mathrm{GPa}, \mathrm{Em}=70 \mathrm{GPa}$, $a / h=10)$

Tables 1, 2 and 3 respectively display the values for non-dimensional deflection, normal stress and shear stress. Results obtained based on a polynomial four variable refined plate theory in this study is compared with those given by Zenkour (2005) based on first [3a] and third [3b] order plate theory, Bessaim et al. (2013) based on high-order shear and normal deformation theory, Nguyen et al. (2014) based on an inverse trigonometric shear deformation theory, Thai et al. (2016) based on a simple four-unknown shear and normal deformations theory. In Table 1, it is seen that the lowest deflection value is obtained with the $p=0$ where the plate is completely ceramic. Its metallic properties increase with the increase in the $\mathrm{p}$ and thus, the deflection increases. Different thickness ratios reveal that the highest deflection value is observed in the (1-0-1) FG sandwich plate where there is no core. The deflection value decreases as the core-to-plate thickness ratio increases. The lowest deflection value is obtained in (1-2-1) FG sandwich plate. Non-dimensional normal stress values are listed in Table 2 with different $\mathrm{p}$ values for different thickness ratios. Nondimensional shear stress values are listed in Table 3 . In the table, obtained results are overassumed compared to the references.

Table 1 - Non-dimensional center deflections of $\mathrm{Al} / \mathrm{ZrO}_{2}$ square sandwich plates.

\begin{tabular}{llllllll}
\hline P & Theory & $1-0-1$ & $2-1-2$ & $2-1-1$ & $1-1-1$ & $2-2-1$ & $1-2-1$ \\
\hline 0 & {$[3 \mathrm{a}]$} & 0.19607 & 0.19607 & 0.19607 & 0.19607 & 0.19607 & 0.19607 \\
& {$[3 \mathrm{~b}]$} & 0.19606 & 0.19606 & 0.19606 & 0.19606 & 0.19606 & 0.19606 \\
& {$[30]$} & - & 0.19486 & 0.19486 & 0.19486 & 0.19486 & 0.19486 \\
& {$[14]$} & 0.19597 & 0.19597 & 0.19597 & 0.19597 & 0.19597 & 0.19597 \\
& {$[31]$} & 0.19490 & 0.19490 & 0.19490 & 0.19490 & 0.19490 & 0.19490 \\
& Present & 0.19606 & 0.19606 & 0.19606 & 0.19606 & 0.19606 & 0.19606 \\
1 & {$[3 a]$} & 0.32484 & 0.30750 & - & 0.29301 & 0.28168 & 0.27167 \\
\hline
\end{tabular}


Table 1 - Non-dimensional center deflections of $\mathrm{Al} / \mathrm{ZrO} \mathrm{O}_{2}$ square sandwich plates.(continue)

\begin{tabular}{|c|c|c|c|c|c|c|c|}
\hline \multirow[t]{6}{*}{$\mathrm{P}$} & Theory & $1-0-1$ & $2-1-2$ & $2-1-1$ & $1-1-1$ & $2-2-1$ & $1-2-1$ \\
\hline & {$[3 \mathrm{~b}]$} & 0.32358 & 0.30632 & - & 0.29199 & 0.28085 & 0.27094 \\
\hline & [30] & - & 0.30430 & 0.29448 & 0.29007 & 0.27874 & 0.26915 \\
\hline & [14] & 0.32348 & 0.30622 & 0.29666 & 0.29191 & 0.28077 & 0.27086 \\
\hline & [31] & 0.32000 & 0.30280 & - & 0.28870 & 0.27780 & 0.26830 \\
\hline & Present & 0.34539 & 0.32677 & 0.31494 & 0.31032 & 0.29665 & 0.28519 \\
\hline \multirow[t]{6}{*}{2} & {$[3 a]$} & 0.37514 & 0.35408 & - & 0.33441 & 0.31738 & 0.30370 \\
\hline & {$[3 \mathrm{~b}]$} & 0.37335 & 0.35231 & - & 0.33289 & 0.31617 & 0.30263 \\
\hline & [30] & - & 0.35001 & 0.33495 & 0.33068 & 0.31356 & 0.30060 \\
\hline & [14] & 0.37322 & 0.35221 & 0.33769 & 0.33279 & 0.31608 & 0.30255 \\
\hline & [31] & 0.36890 & 0.34740 & - & 0.32830 & 0.31190 & 0.29900 \\
\hline & Present & 0.38650 & 0.36655 & 0.35030 & 0.34653 & 0.32763 & 0.31366 \\
\hline \multirow[t]{6}{*}{5} & {$[3 a]$} & 0.41120 & 0.39418 & - & 0.37356 & 0.35123 & 0.33631 \\
\hline & {$[3 \mathrm{~b}]$} & 0.40927 & 0.39183 & - & 0.37145 & 0.34960 & 0.33480 \\
\hline & [30] & - & 0.38934 & 0.36981 & 0.36902 & 0.34649 & 0.33255 \\
\hline & [14] & 0.40911 & 0.39170 & 0.37295 & 0.37134 & 0.34950 & 0.33472 \\
\hline & [31] & 0.40530 & 0.38630 & - & 0.36580 & 0.34450 & 0.33040 \\
\hline & Present & 0.41291 & 0.39706 & 0.39760 & 0.37727 & 0.35452 & 0.33994 \\
\hline \multirow[t]{6}{*}{10} & {$[3 a]$} & 0.41919 & 0.40657 & - & 0.38787 & 0.36395 & 0.34996 \\
\hline & {$[3 b]$} & 0.41772 & 0.40407 & - & 0.38551 & 0.36215 & 0.34824 \\
\hline & [30] & - & 0.40153 & 0.38111 & 0.38303 & 0.35885 & 0.34591 \\
\hline & [14] & 0.41754 & 0.40398 & 0.38430 & 0.38540 & 0.36202 & 0.34815 \\
\hline & [31] & 0.41450 & 0.39890 & - & 0.38010 & 0.35740 & 0.34430 \\
\hline & Present & 0.41900 & 0.41295 & 0.39248 & 0.39482 & 0.37041 & 0.35683 \\
\hline
\end{tabular}

Table 2 - Non-dimensional normal stress of $\mathrm{Al} / \mathrm{ZrO} \mathrm{O}_{2}$ square sandwich plates.

\begin{tabular}{|c|c|c|c|c|c|c|c|}
\hline $\mathrm{P}$ & Theory & $1-0-1$ & $2-1-2$ & $2-1-1$ & $1-1-1$ & $2-2-1$ & $1-2-1$ \\
\hline \multirow[t]{6}{*}{0} & [3a] & 1.97576 & 1.97576 & 1.97576 & 1.97576 & 1.97576 & 1.97576 \\
\hline & {$[3 \mathrm{~b}]$} & 2.04985 & 2.04985 & 2.04985 & 2.04985 & 2.04985 & 2.04985 \\
\hline & {$[30]$} & - & 1.99524 & 1.99524 & 1.99524 & 1.99524 & 1.99524 \\
\hline & [14] & 1.99482 & 1.99482 & 1.99482 & 1.99482 & 1.99482 & 1.99482 \\
\hline & [31] & 2.00480 & 2.00430 & - & 2.00400 & 2.00340 & 2.00360 \\
\hline & Present & 1.95721 & 1.95721 & 1.95721 & 1.95721 & 1.95721 & 1.95721 \\
\hline
\end{tabular}


Static Analysis of Simply Supported Functionally Graded Sandwich Plates by ...

Table 2 - Non-dimensional normal stress of $\mathrm{Al} / \mathrm{ZrO}_{2}$ square sandwich plates.(continue)

\begin{tabular}{|c|c|c|c|c|c|c|c|}
\hline $\mathrm{P}$ & Theory & $1-0-1$ & $2-1-2$ & $2-1-1$ & $1-1-1$ & $2-2-1$ & $1-2-1$ \\
\hline \multirow[t]{6}{*}{1} & [3a] & 1.53245 & 1.45167 & - & 1.38303 & 1.27749 & 1.28096 \\
\hline & {$[3 \mathrm{~b}]$} & 1.57923 & 1.49587 & - & 1.42617 & 1.32062 & 1.32309 \\
\hline & {$[30]$} & - & 1.46131 & 1.35053 & 1.39243 & 1.28274 & 1.29030 \\
\hline & [14] & 1.54441 & 1.46297 & 1.35703 & 1.39406 & 1.28852 & 1.29174 \\
\hline & [31] & 1.56760 & 1.48570 & - & 1.41520 & 1.30280 & 1.30970 \\
\hline & Present & 1.62848 & 1.54457 & 1.41548 & 1.46713 & 1.33792 & 1.34633 \\
\hline \multirow[t]{6}{*}{2} & {$[3 a]$} & 1.77085 & 1.67496 & - & 1.58242 & 1.42528 & 1.43580 \\
\hline & {$[3 b]$} & 1.82167 & 1.72144 & - & 1.62748 & 1.47095 & 1.47988 \\
\hline & {$[30]$} & - & 1.68472 & 1.52101 & 1.59170 & 1.42887 & 1.44497 \\
\hline & [14] & 1.78383 & 1.68682 & 1.52988 & 1.59393 & 1.43693 & 1.44707 \\
\hline & [31] & 1.81230 & 1.71730 & - & 1.62320 & 1.45640 & 1.47210 \\
\hline & Present & 1.82102 & 1.73586 & 1.56265 & 1.64304 & 1.46762 & 1.48584 \\
\hline \multirow[t]{6}{*}{5} & {$[3 \mathrm{a}]$} & 1.93576 & 1.86479 & - & 1.76988 & 1.56401 & 1.59309 \\
\hline & {$[3 \mathrm{~b}]$} & 1.99272 & 1.91302 & - & 1.81580 & 1.61181 & 1.63814 \\
\hline & [30] & - & 1.87516 & 1.66856 & 1.77919 & 1.56627 & 1.60203 \\
\hline & [14] & 1.95031 & 1.87709 & 1.67895 & 1.78159 & 1.57620 & 1.60459 \\
\hline & [31] & 1.97620 & 1.91190 & - & 1.81800 & 1.60150 & 1.63830 \\
\hline & Present & 1.93514 & 1.87915 & 1.67627 & 1.79068 & 1.57722 & 1.61390 \\
\hline \multicolumn{2}{|c|}{$10[3 \mathrm{a}]$} & 1.96780 & 1.92165 & - & 1.83754 & 1.61645 & 1.65844 \\
\hline \multicolumn{2}{|c|}{ [3b] } & 2.03036 & 1.97126 & - & 1.88376 & 1.66660 & 1.70417 \\
\hline \multicolumn{2}{|r|}{ [30] } & - & 1.93266 & 1.71835 & 1.84705 & 1.61792 & 1.66754 \\
\hline \multicolumn{2}{|r|}{ [14] } & 1.98382 & 1.93431 & 1.72890 & 1.84933 & 1.62840 & 1.67019 \\
\hline \multicolumn{2}{|r|}{ [31] } & 2.00410 & 1.96840 & - & 1.88820 & 1.65820 & 1.70990 \\
\hline \multicolumn{2}{|r|}{ Present } & 1.95453 & 1.91930 & 1.71255 & 1.84092 & 1.61617 & 1.66440 \\
\hline
\end{tabular}

Figure 3 shows the non-dimensional deflection of the symmetric sandwich plates with different thickness ratios. In each of four symmetric ratios, the lowest maximum deflection value is obtained for $p=0$ as expected. It is observed that, maximum deflection is increased along with increasing power convention index. When all the four symmetric ratios are compared, it is seen that, the highest maximum deflection value is obtained in the (1-0-1) FG sandwich plate. The second largest maximum deflection after the (1-0-1) FG sandwich plate occurs in the (2-1-2) FG sandwich plate, which is the condition where face sheet thickness is the highest as compared to the core thickness. In the (1-1-1) and (1-2-1) FG sandwich plates whose face thicknesses are equal, it is observed that the maximum deflection value decreases with the increase in the thickness of the core. Also, the non-dimensional deflection of the 
non-symmetric sandwich plates with two different thickness ratios are given in the Figure 3. In the (2-2-1) FG sandwich plate where the core is thicker, the maximum deflection has a lower value. In the (2-1-1) FG sandwich plate, it is observed that the maximum deflection rapidly increases with increase in the $\mathrm{p}$ value.

Table 3 - Non-dimensional shear stress of $\mathrm{Al} / \mathrm{ZrO} \mathrm{O}_{2}$ square sandwich plates.

\begin{tabular}{|c|c|c|c|c|c|c|c|}
\hline $\mathrm{P}$ & Theory & $1-0-1$ & $2-1-2$ & $2-1-1$ & $1-1-1$ & $2-2-1$ & $1-2-1$ \\
\hline \multirow[t]{6}{*}{0} & {$[3 a]$} & 0.19099 & 0.19099 & 0.19099 & 0.19099 & 0.19099 & 0.19099 \\
\hline & {$[3 b]$} & 0.23857 & 0.23857 & - & 0.23857 & 0.23857 & 0.23857 \\
\hline & [30] & - & 0.23794 & 0.23794 & 0.23794 & 0.23794 & 0.23794 \\
\hline & [14] & 0.23581 & 0.23581 & 0.23581 & 0.23581 & 0.23581 & 0.23581 \\
\hline & [31] & 0.19510 & 0.19510 & - & 0.19510 & 0.19480 & 0.19510 \\
\hline & Present & 0.23852 & 0.23852 & 0.23852 & 0.23852 & 0.23852 & 0.23852 \\
\hline \multirow[t]{6}{*}{1} & {$[3 \mathrm{a}]$} & 0.26099 & 0.24316 & - & 0.23257 & 0.22762 & 0.22057 \\
\hline & {$[3 b]$} & 0.29203 & 0.27104 & - & 0.26117 & 0.25951 & 0.25258 \\
\hline & [30] & - & 0.27050 & 0.27017 & 0.26060 & 0.25890 & 0.25196 \\
\hline & [14] & 0.28953 & 0.26882 & 0.26852 & 0.25906 & 0.25736 & 0.25054 \\
\hline & [31] & 0.29810 & 0.28050 & - & 0.26820 & 0.25910 & 0.25190 \\
\hline & Present & 0.30078 & 0.26934 & 0.26824 & 0.25440 & 0.25222 & 0.24166 \\
\hline \multirow[t]{6}{*}{2} & {$[3 a]$} & 0.29731 & 0.26752 & - & 0.25077 & 0.24316 & 0.23257 \\
\hline & {$[3 b]$} & 0.32622 & 0.28838 & - & 0.27188 & 0.26939 & 0.25834 \\
\hline & [30] & - & 0.28792 & 0.28742 & 0.27138 & 0.26885 & 0.25776 \\
\hline & [14] & 0.32336 & 0.28607 & 0.28569 & 0.26982 & 0.26731 & 0.25645 \\
\hline & [31] & 0.34090 & 0.31520 & - & 0.29750 & 0.28410 & 0.27420 \\
\hline & Present & 0.34187 & 0.28911 & 0.28651 & 0.26543 & 0.26153 & 0.24566 \\
\hline \multirow[t]{6}{*}{5} & {$[3 \mathrm{a}]$} & 0.34538 & 0.29731 & - & 0.27206 & 0.26009 & 0.24596 \\
\hline & {$[3 \mathrm{~b}]$} & 0.38634 & 0.31454 & - & 0.28643 & 0.28265 & 0.26512 \\
\hline & [30] & - & 0.31419 & 0.31293 & 0.28606 & 0.28217 & 0.26463 \\
\hline & [14] & 0.38250 & 0.31182 & 0.31087 & 0.28420 & 0.28047 & 0.26327 \\
\hline & [31] & 0.38260 & 0.34920 & - & 0.32660 & 0.30900 & 0.29690 \\
\hline & Present & 0.40619 & 0.31798 & 0.31239 & 0.28156 & 0.27490 & 0.25209 \\
\hline \multirow[t]{6}{*}{10} & {$[3 a]$} & 0.37277 & 0.31316 & - & 0.28299 & 0.26998 & 0.25257 \\
\hline & {$[3 \mathrm{~b}]$} & 0.43206 & 0.33242 & - & 0.29566 & 0.29080 & 0.26895 \\
\hline & [30] & - & 0.33210 & 0.32959 & 0.29534 & 0.29036 & 0.26850 \\
\hline & [14] & 0.42744 & 0.32936 & 0.32732 & 0.29326 & 0.28854 & 0.26705 \\
\hline & [31] & 0.40020 & 0.36680 & - & 0.33960 & 0.32040 & 0.30770 \\
\hline & Present & 0.44818 & 0.33540 & 0.32754 & 0.29172 & 0.28274 & 0.25611 \\
\hline
\end{tabular}




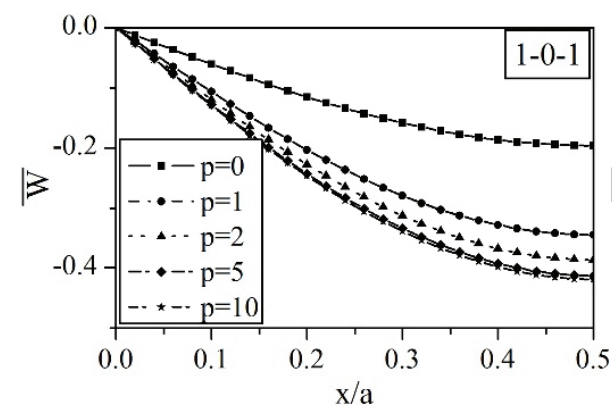

(a) (1-0-1) FG sandwich plate

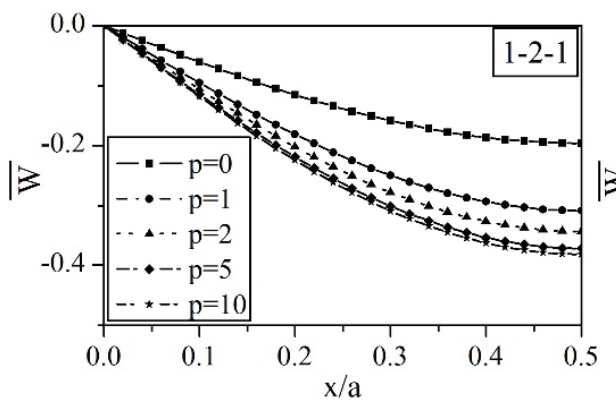

(c) (1-2-1) FG sandwich plate

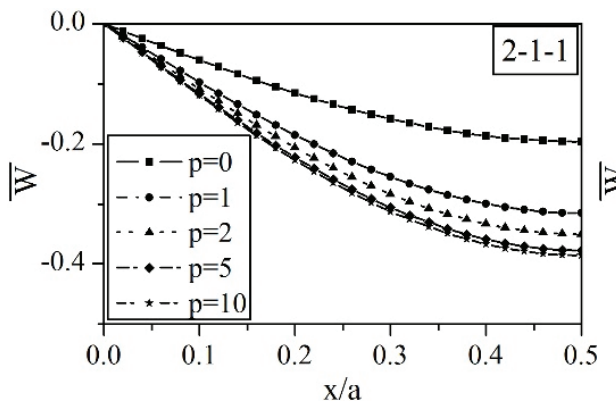

(e) (2-1-1) FG sandwich plate

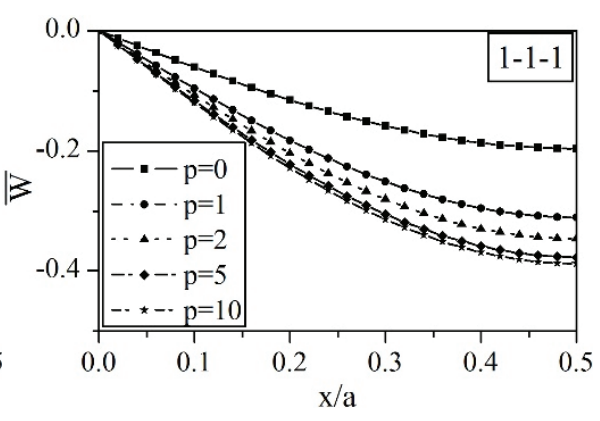

(b) (1-1-1) FG sandwich plate

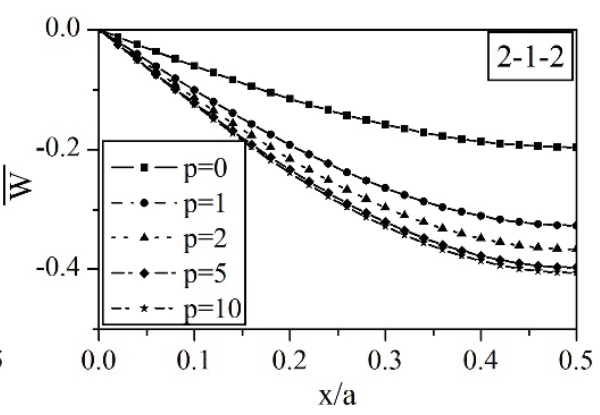

(d) (2-1-2) FG sandwich plate

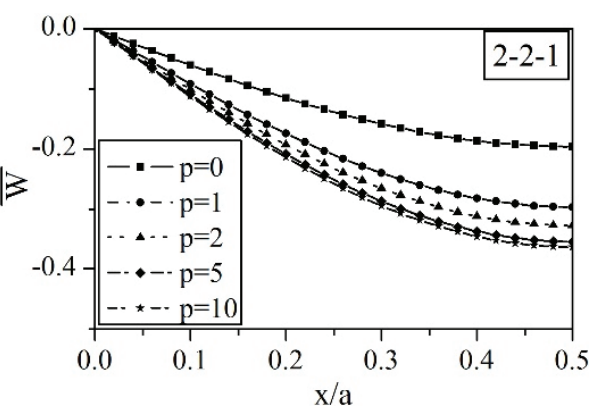

(f) (2-2-1) FG sandwich plate

Fig.3 - Nondimensional deflection of the symmetric and nonsymmetric square sandwich plates with varying thickness combinations

In the Figure 4, non-dimensional normal stress distributions of the symmetric sandwich plates along the thickness are given. In the (1-0-1) FG sandwich plate, the normal stress shows a linear distribution for $\mathrm{p}=0$ and a parabolic distribution with the increase in $\mathrm{p}$, and the values for maximum tensile and compressive stress decrease. In the (1-1-1) FG sandwich plate, which has core and face sheets thicknesses of equal value, it is observed that the local extreme stress, which occurs between the face and the core, is lower than the (2-1-2) FG plate. Nevertheless, it is clear that the highest local extreme stress occurs in the (1-2-1) FG 
sandwich plate. For all thickness ratios, maximum tensile stress and compressive stress values decrease with the increase in the power convention index. Figure 4 also presents the non-dimensional normal stress distributions of the non-symmetric sandwich plates along the thickness. The normal stress distribution of the core appears to be linear for both plates, and the stress, which occurs between the face sheet and the interface of the core in the (2-1-1) FG sandwich plate is lower. The stress value decreases in the thinner face sheet with the increase in p. In the (2-2-1) FG sandwich plate, it is observed that the normal stress, which occurs between the thin face and core interface, is higher in comparison with the (2-1-1) FG sandwich plate.

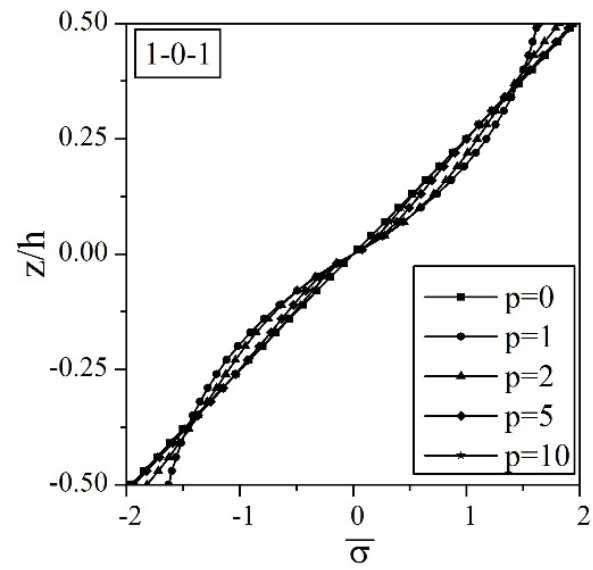

(a) (1-0-1) FG sandwich plate

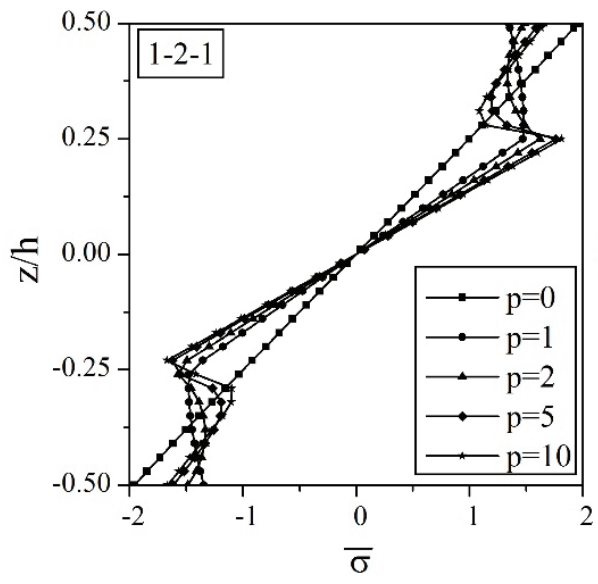

(c) (1-2-1) FG sandwich plate

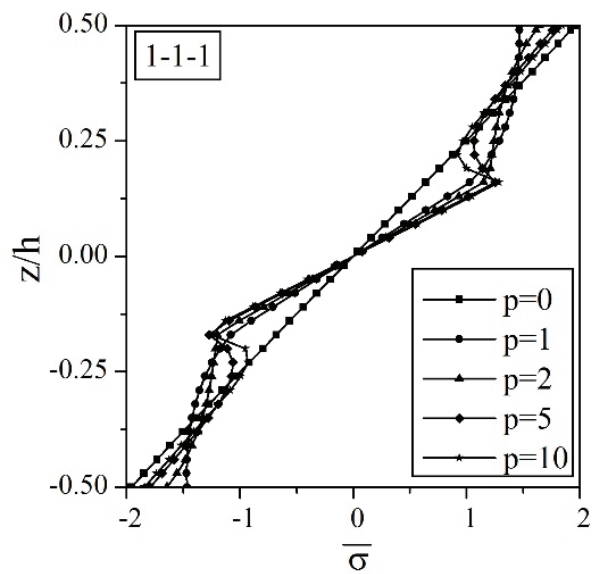

(b) (1-1-1) FG sandwich plate

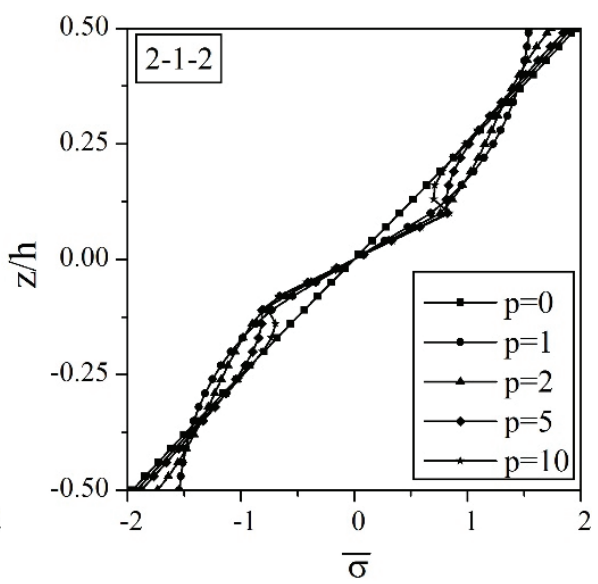

(d) (2-1-2) FG sandwich plate 


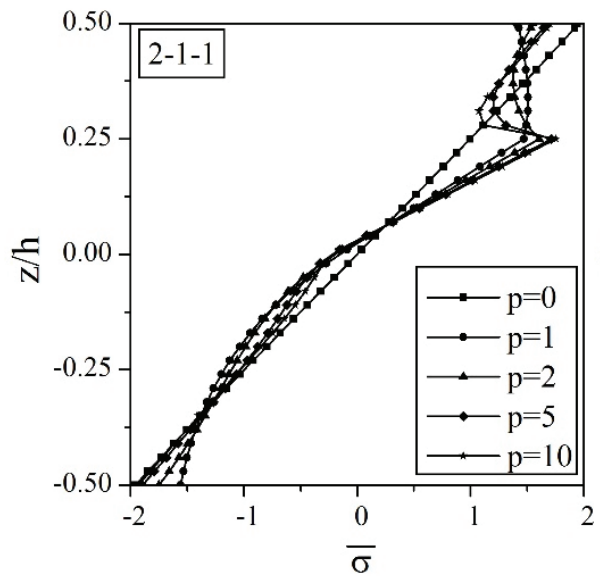

(e) (2-1-1) FG sandwich plate

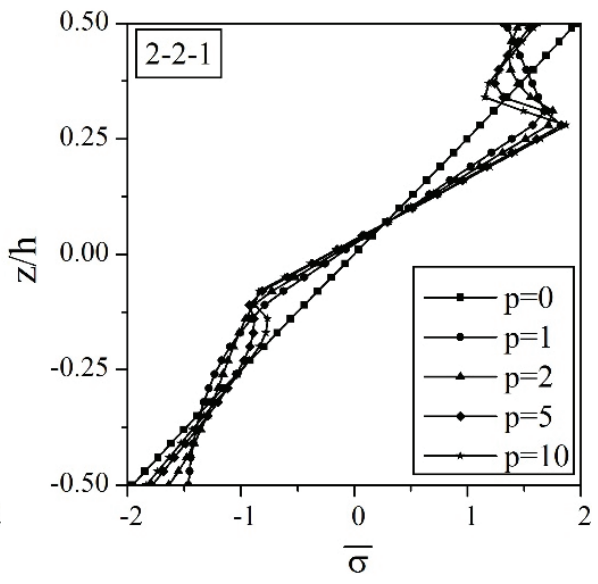

(f) (2-2-1) FG sandwich plate

Fig.4 - Non-dimensional Normal Stress for different $p$ values for symmetric and nonsymmetric square sandwich plates.

Figure 5 shows the normal stress distributions along the thickness direction for different ratios of core and face sheet thickness values. Figure 5(a) demonstrates $p=1$ and Figure 5(b) demonstrates $p=5$. The highest normal stress values are seen at the interface of the core and face sheets in (1-2-1) FG sandwich plate for both $p=1$ and $p=5$. For $p=5$, local extreme stresses are observed at the interfaces.
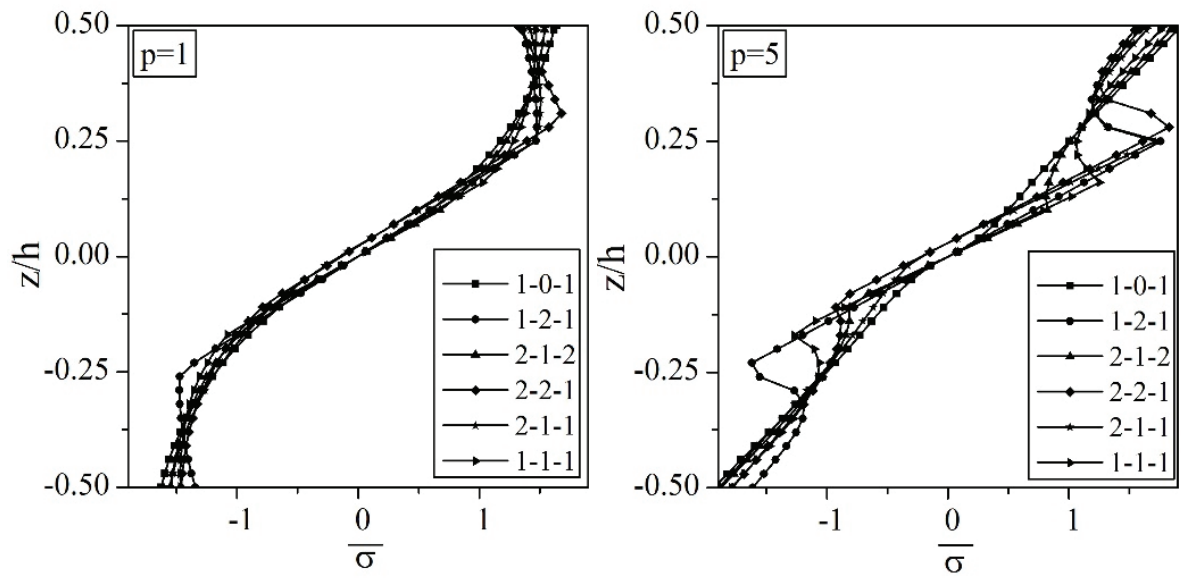

Fig.5 - Dimensionless Normal Stress for different type of square sandwich plates.

Shear stress distributions of the symmetric sandwich plates along the thickness direction for different power law index values are seen in Figure 6. In (1-0-1) FG sandwich plate, which does not contain a core, shows that the maximum, stress which occurs in the interface between lower and upper face sheets, rapidly increases with the increase in the p-value. In 
the (1-1-1) FG sandwich plate, in which the thickness of the core and the face are equal, it is observed that the shear stress value in the face sheets decreases, but the shear stress value at interface of the core and the face sheets, and the maximum shear stress increases with increasing p-value. With the increase in the p-value in the (1-2-1) FG sandwich plate, in which the thickness of the core is higher than that of the face sheets, the shear stress value decreases in the face sheets, but the same value slightly increases in the core. In the (2-1-2) FG sandwich plate, which has the smallest core-to-face thickness ratio, the shear stress decreases with the increasing $p$-value, but the shear stress values of the core and the interface increase rapidly. Shear stress distributions of the non-symmetric sandwich plates along the thickness direction for the power convention index values can be seen in Figure 6. In both combinations, the shear stress of the core has a non-symmetric distribution. The shear stress, which occurs in the interface between the core and the thicker face sheet is considerably higher than the other interface. In the shear stress distribution that occurs in the face sheets it can be seen that the thin face sheet is less severely affected by the power convention index, and the stress distribution of the thicker face sheet decreases with the increasing $p$-value in both conditions.

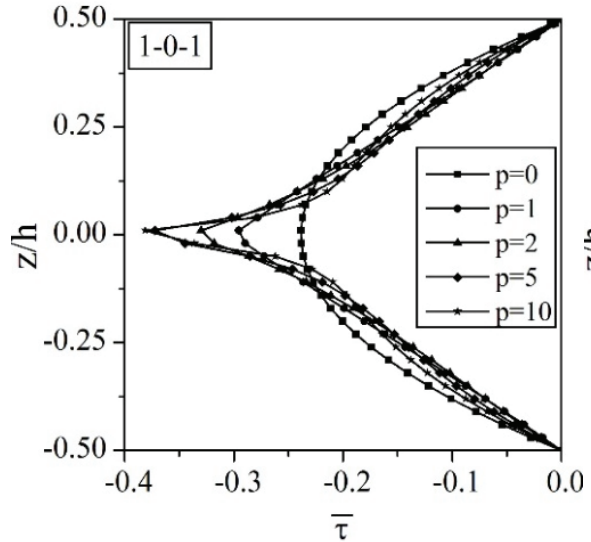

(a) (1-0-1) FG sandwich plate

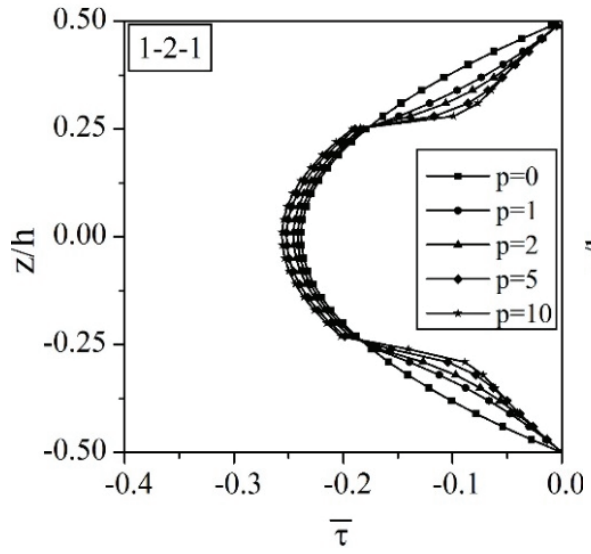

(c) (1-2-1) FG sandwich plate

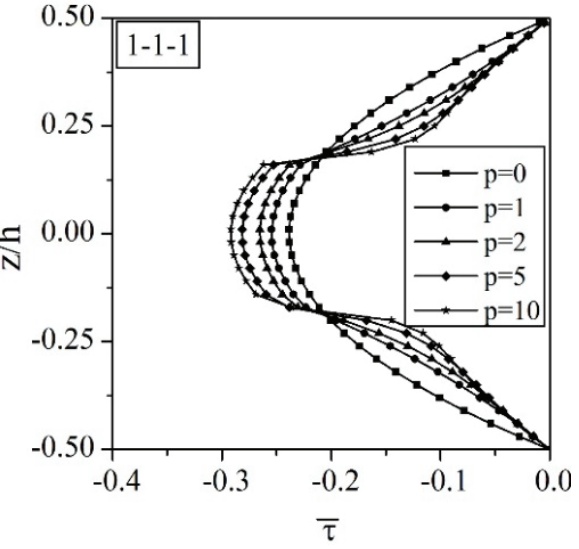

(b) (1-1-1) FG sandwich plate

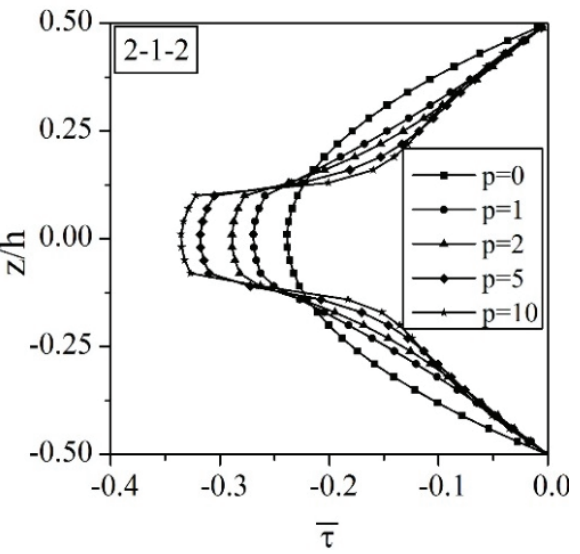

(d) (2-1-2) FG sandwich plate 


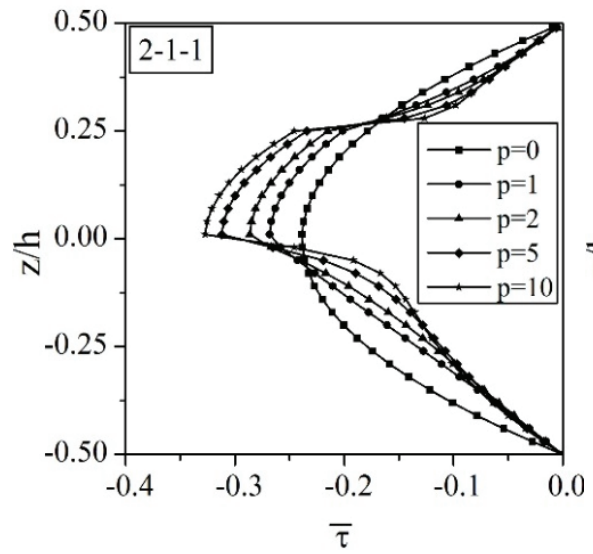

(e) (2-1-1) FG sandwich plate

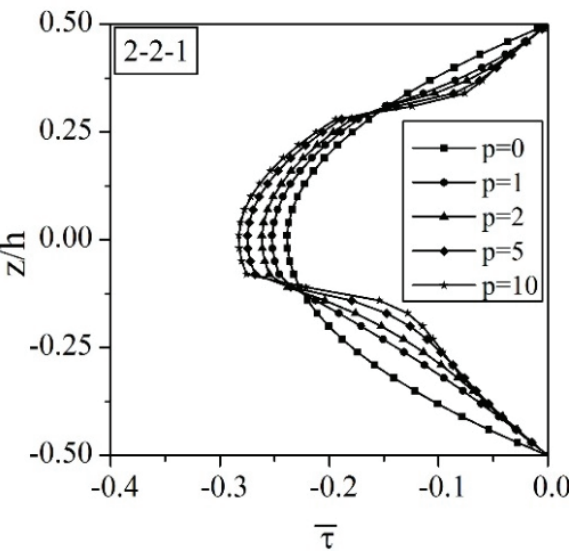

(f) (2-2-1) FG sandwich plate

Fig.6 - Non-dimensional Shear Stress for different $p$ values for symmetric and nonsymmetric square sandwich plates.

Figure 7 gives the shear stress distributions along the thickness direction for different ratios of core and face sheet thickness values. Figure 7(a) demonstrates $p=1$ and Figure 7(b) demonstrates $p=5$. The shear stress in all ratios increases with the increase in the $p$-value. In the shear stress distribution of the (1-0-1) FG sandwich plate for $p=5$, it is observed that the shear stress rapidly increases in the section closer to the interfaces, and it takes values that are very similar to the $\mathrm{p}=2$ condition in the areas closer to the face sheets. The stress distributions remain in the same form with the increase in the p-value and they are shifted towards higher values. The analysis of the shear stress distributions of the sandwiches with symmetric combinations reveals that as the core-to-face thickness ratio increases, the maximum shear stress and the shear stress which occurs in the interfaces decrease.
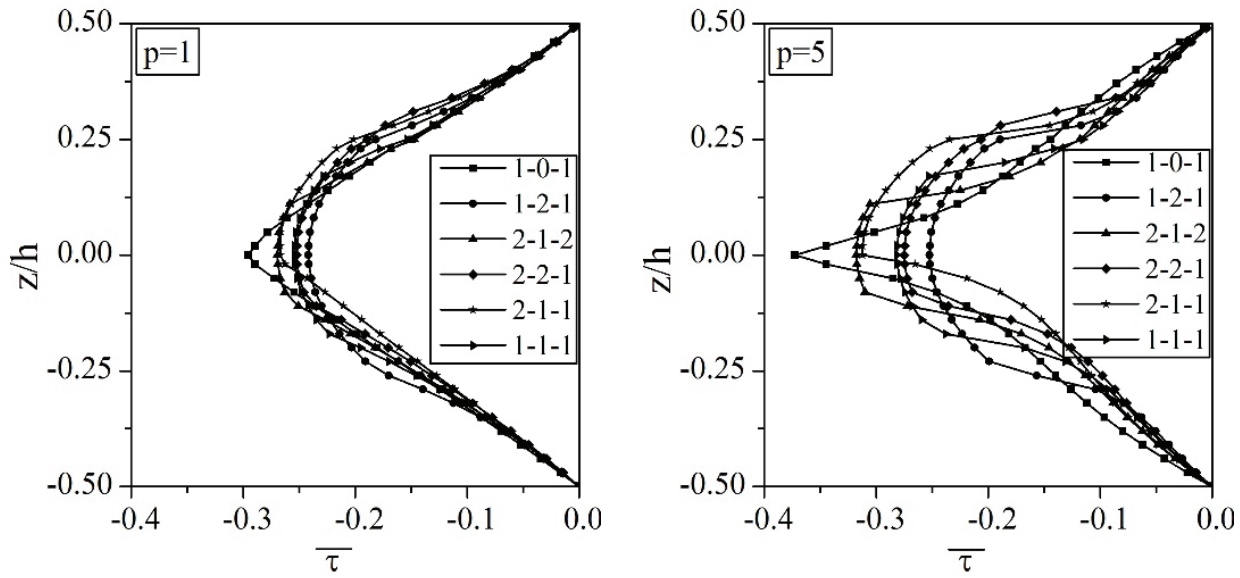

Fig.7 - Dimensionless transverse shear stress for different type of square sandwich plates. 


\section{CONCLUSION}

Static analysis of a functionally graded square sandwich plate is studied in this paper. The effective properties of the functionally graded face sheets are varied through thickness direction with the Mori-Tanaka Micromechanical Model. For displacement fields, the Four Variable Plate Theory is used. The theory separates the bending and shear coupling of vertical displacements, so it is very simple and accurate. The equations of the simply supported square plate are obtained through the virtual displacement principle. Navier's approach is used for the closed-form solution. Numerical results are obtained for $\mathrm{Al}-\mathrm{ZrO}_{2}$ square sandwich plate with sinusoidal loading. Non-dimensional deflection, normal stress and shear stress values for different core and face sheet thickness ratios are obtained and compared with the references. It is seen that obtained results are sufficiently in agreement with the other theories.

\section{References}

[1] Reddy, J.N., Wang, C.M., An overview of the relationships between solutions of the classical and shear deformation plate theories. Composites Science and Technology, 60, 2327-2335, 2000.

[2] Thai, H.T., Kim, S.E., A review of theories for the modeling and analysis of functionally graded plates and shells. Composite Structures. 128, 70-86, 2015.

[3] Zenkour, A.M., A comprehensive analysis of functionally graded sandwich plates: Part 1-Deflection and stresses. International Journal of Solids and Structures, 42, 5224$5242,2005$.

[4] Zenkour, A.M., Alghamdi, N.A., Bending analysis of functionally graded sandwich plates under the effect of mechanical and thermal loads. Mechanics of Advanced Materials and Structures, 17, 419-432, 2010.

[5] Thai, H.T., Choi, D.H., A simple first-order shear deformation theory for the bending and free vibration analysis of functionally graded plates. Composite Structures, 101, 332-340, 2013.

[6] Thai, H.T., Nguyen, T.K., Vo, T.P., Lee, J., Analysis of functionally graded sandwich plates using a new first-order shear deformation theory. European Journal of Mechanics A/Solids, 45, 211-225, 2014.

[7] Kim, J., Reddy, J.N., Analytical solutions for bending, vibration, and buckling of FGM plates using a couple stress-based third-order theory. Composite Structures, 103, 8698, 2013.

[8] Tounsi, A., Houari, M.S.A., Benyoucef, S., Bedia, E.A.A., A refined trigonometric shear deformation theory for thermoelastic bending of functionally graded sandwich plates. Aerospace Science and Technology, 24, 209-220, 2013.

[9] Reddy, J.N., Analysis of functionally graded plates. International Journal for Numerical Methods in Engineering, 47, 663-684, 2000. 
[10] Mantari, J.L., Oktem, A.S., Soares, C.G., Static and dynamic analysis of laminated composite and sandwich plates and shells by using a new higher-order shear deformation theory. Composite Structures, 94, 37-49, 2011.

[11] Abdelaziz, H.H., Atmane, H.A., Mechab, I., Boumia, L., Tounsi, A., Abbas, A.B.E., Static analysis of functionally graded sandwich plates using an efficient and simple refined theory. Chinese Journal of Aeronautics, 24, 434-448, 2011.

[12] Mantari, J.L., Granados, E.V., Thermoelastic analysis of advanced sandwich plates based on a new quasi-3D hybrid type HSDT with 5 unknowns. Composites: Part B, 69, 317-334, 2014.

[13] Brischetto, S., Leetsch, R., Carrera, E., Wallmersperger, T., Kröplin, B., Thermomechanical bending of functionally graded plates. Journal of Thermal Stresses, 31, 286308, 2008.

[14] Nguyen, V.H., Nguyen, T.K., Thai, H.T., Vo, T.P., A new inverse trigonometric shear deformation theory for isotropic and functionally graded sandwich plates. Composites: Part B, 66, 233-246, 2014.

[15] Li, X.F., Wang, B.L., Han, J.C., A higher-order theory for static and dynamic analyses of functionally graded beams. Archive of Applied Mechanics, 80, 1197-1212, 2010.

[16] Akavci, S.S., Tanrikulu, A.H., Static and free vibration analysis of functionally graded plates based on a new quasi-3D and 2D shear deformation theories. Composites Part B, 83, 203-215, 2015.

[17] Shimpi, R.P., Refined Plate Theory and Its Variants. AIAA JOURNAL, 40, 137-146, 2002.

[18] Mechab, I., Atmane, H.A., Tounsi, A., Belhadj, H.A., Bedia, E.A.A., A two variable refined plate theory for the bending analysis of functionally graded plates. Acta Mechanica Sinica, 26, 941-949, 2010.

[19] Houari, M.S.A., Benyoucef, S., Mechab, I., Tounsi, A., Bedia, E.A.A., Two-variable refined plate theory for thermoelastic bending analysis of functionally graded sandwich plates. Journal of Thermal Stresses, 34(4), 315-334, 2011.

[20] Benachour, A., Tahar, H.D., Atmane, H.A., Tounsi, A., Ahmed, M.S., A four variable refined plate theory for free vibrations of functionally graded plates with arbitrary gradient. Composites Part B-Engineering, 42(6), 1386-1394, 2011.

[21] Hadji, L., Atmane, H.A., Tounsi, A., Mechab, I., Bedia, E.A., Free vibration of functionally graded sandwich plates using four-variable refined plate theory. Applied Mathematics and Mechanics, 32(7), 925-942, 2011.

[22] Bouiadjra, M.B., Houari, A.M.S., Tounsi, A., Thermal buckling of functionally graded plates according to a four-variable refined plate theory. Journal of Thermal Stresses, 35(8), 677-694, 2012.

[23] Demirhan, P.A., Taskin, V., Levy solution for bending analysis of functionally graded sandwich plates based on four variable plate theory. Composite Structures, 177, 80-95, 2017. https://doi.org/10.1016/j.compstruct.2017.06.048. 
[24] Thai, H.T., Choi, D.H., A refined shear deformation theory for free vibration of functionally graded plates on elastic foundation. Composites Part B-Engineering, 43(5), 2335-2347, 2012.

[25] Thai, H.T., Uy, B., Levy solution for buckling analysis of functionally graded plates based on a refined plate theory. Proceedings of The Institution of Mechanical Engineers Part C-journal of Mechanical Engineering Science, 227(12), 2649-2664, 2013.

[26] Thai, H.T., Choi, D.H., Improved refined plate theory accounting for effect of thickness stretching in functionally graded plates. Composites: Part B, 56, 705-716, 2014.

[27] Demirhan, P.A., Taskin, V., Static analysis of simply supported foam core sandwich plate, Materials, Methods \& Technologies, ISSN 1314-7269, Volume 10, 2016.

[28] Thai, H.T., Choi, D.H., Levy solution for free vibration analysis of functionally graded plates based on a refined plate theory. KSCE Journal of Civil Engineering 18(6), 18131824, 2014.

[29] Rouzegar, J., Abad, F., Free vibration analysis of FG plate with piezoelectric layers using four-variable refined plate theory. Thin-Walled Structures, 89, 76-83, 2015.

[30] Bessaim, A., Houari, M.S.A., Tounsi, A., Mahmoud, S.R., Bedia, E.A.A., A new higher-order shear and normal deformation theory for the static and free vibration analysis of sandwich plates with functionally graded isotropic face sheets. Journal of Sandwich Structures and Materials, 15(6), 671-703, 2013.

[31] Thai, C.H., Zenkour, A.M., Wahab, M.A., Nguyen-Xuan, H., A simple four-unknown shear and normal deformations theory for functionally graded isotropic and sandwich plates based on isogeometric analysis. Composite Structures, 139, 77-95, 2016. 
